\title{
Guidelines of the Italian Society of Videosurgery (SIVI) in Infancy for the minimally invasive treatment of Hypertrophic Pyloric Stenosis in neonates and infants
}

\author{
Salvatore Fabio Chiarenza, ${ }^{1}$ Cosimo Bleve, ${ }^{1}$ Maria Escolino, ${ }^{2}$ Ciro Esposito, ${ }^{2}$ Fabio Beretta, ${ }^{3}$ \\ Maurizio Cheli, ${ }^{4}$ Maria Grazia Scuderi, 5 Vincenzo Di Benedetto, ${ }^{5}$ Giovanni Casadio, ${ }^{6}$ Maurizio Marzaro, ${ }^{7}$ \\ Marco Gambino, ${ }^{8}$ Andrea Conforti, ${ }^{9}$ Alessio Pini Prato, ${ }^{10}$ Francesco Molinaro, ${ }^{11}$ Simona Gerocarni Nappo, ${ }^{12}$ \\ Paolo Caione, ${ }^{13}$ Mario Mendoza-Sagaon 14
}

1 Pediatric Surgery and Urologic Unit, Regional Center of Minimally Invasive Surgery and Urology, S. Bortolo Hospital, Vicenza; ${ }^{2}$ Pediatric Surgery Unit, Federico II University, Naples; ${ }^{3}$ Pediatric Surgery Unit, APSS, Trento; ${ }^{4}$ Pediatric Surgery Department Papa Giovanni XXIII Hospital, Bergamo; 5 Pediatric Surgery Unit, Vittorio Emanuele Hospital, Catania; ' $P$ Pediatric Surgery Unit, Parma University Hospital, Parma; ${ }^{7}$ Pediatric Surgery Unit, Local Health Unit 2, Treviso Hospital, Treviso; ${ }^{8}$ Pediatric Surgery Unit, Annunziata Civil Hospital, Cosenza; ${ }^{9}$ Congenital Esophageal Disorders Unit, Neonatal Surgery Unit, Bambino Gesù Children's Research Hospital, Rome; 10Unit of Pediatric Surgery, The Children Hospital, Azienda Ospedaliera SS Antonio e Biagio e Cesare Arrigo, Alessandria; ${ }^{11}$ Department of Medical Sciences, Surgery and Neuroscience, Section of Pediatric Surgery, University of Siena, Siena; 12 Pediatric Surgery Unit, Regina Margherita Hospital, Turin; 13Pediatric Surgery and Urologic Unit, Pediatric Hospital Bambino Gesù, Rome, Italy; ${ }^{14}$ Service of Paediatric Surgery and Paediatric Orthopaedics, Regional Hospital of Bellinzona, Switzerland

\section{Background}

The SIVI (Italian Society of Videosurgery in Infancy) guidelines are clinical practice guidelines edited and approved by the Society's steering committee. They are the products of a detailed systematic review of the literature, integrated with expert opinion in the field of pediatric minimally invasive surgery. These guidelines are intended to assist the pediatric surgeons experienced or not experienced in minimally invasive surgery, with the goal to inform choices related to the indications, approach, and techniques to use when treating the major pediatric surgical pathologies.

Given the complexities of congenital malformations and other pediatric surgical conditions, as well as large variations in avail-
Correspondence: Salvatore Fabio Chiarenza, Pediatric Surgery and Urologic Unit, Regional Center of Minimally Invasive Surgery and Urology, S. Bortolo Hospital, viale Rodolfi 37, 36100, Vicenza, Italy. Tel.: +39.0444 .752642 .

Fax: +39.0444 .752643 .

E-mail: fabio.chiarenza@aulss8.veneto.it

Key words: Infantile hypertrophic pyloric stenosis; Laparoscopy; Pyloromyotomy.

Received for publication: 14 October 2020

Accepted for publication: 14 October 2020

This work is licensed under a Creative Commons Attribution NonCommercial 4.0 License (CC BY-NC 4.0).

OCopyright: the Author(s), 2020

Licensee PAGEPress, Italy

La Pediatria Medica e Chirurgica 2020; $42: 243$

doi:10.4081/pmc.2020.243 able regional health services, we must note that these guidelines are not intended as a cookbook recipe to follow for all possible patients. Rather, the guidelines should serve as a flexible framework, to be used by the physician in concert with the parents, to choose the best approach for each individual patient. Decisions tailored to available scientific knowledge and the needs and desires of the patient's family serve both patient autonomy and medical science.

All guidelines are published in this scientific Journal, in order to ensure their availability to all physicians.

The Guidelines project has been approved by the SIVI General Assembly of the 2016 Madrid congress.

Review of guidelines has been performed by the Steering Committee of SIVI and experts.

\section{Introduction}

The most appropriate treatment for the infantile Hypertrophic Pyloric Stenosis (HPS) is still debated. The non-surgical conservative treatment with oral or intravenous administration of atropine does not enjoy a widespread appreciation for several factors: i) the success rate; ii) the necessity of a long-term therapy; iii) the side effects of this latter.1,2 In 1646 Hildanus ${ }^{3,4}$ described the symptoms that were most likely characteristic of a congenital pyloric stenosis and in 1717 Blain published the first autopsy report. Precisely in 1888, Hirschsprung identified the anatomical pathology and the clinical aspects of the condition. ${ }^{5}$ The current surgical solution, the extramucosal pyloromyotomy, was suggested by Dufour and Fredet in $1908^{6}$ and by Weber in 1910, but it was developed by Ramstedt in 1912. The surgical treatment described in 1912 by Dr. Conrad Ramstedt remains the most appropriate standard management option thanks to the higher rate of success $(\sim 100 \%)$, the low level of complications and a shorter length of 
hospital stay. 1,3 Over the past years, the minimally invasive surgery applied to the pediatric field has increasingly gained ground and consideration in the surgical treatment of the HPS although it has generated discussions and disagreements on the advantages, costs, and benefits in comparison to the traditional open surgical approach .7-9

For this reason, it is therefore necessary to write guidelines that could help the pediatric surgeon in the choice of the most adequate approach in performing a "Ramstedt's extramucosal pyloromyotomy" according to the clinical picture, the personal surgical experience and the resources of the healthcare facility.

\section{Definition}

The HPS represents the most common cause of neonatal bowel obstruction and one of the conditions that most frequently requires surgery within the first 2 months of life. The condition is determined by the hypertrophy of the muscle layer (mainly noted for the circular fibers than for the longitudinal ones) of the pylorus with consequent narrowing and elongation of the pyloric canal. The exact etiology is unknown however several studies have suggested a correlation with: genetic factors, ${ }^{10}$ maternal smoking during pregnancy, preterm birth, reduced weight for gestational age, cesarean section, ${ }^{11}$ young maternal age ${ }^{12}$ and exposure to erythromycin during the neonatal period, in particular during the first two weeks after birth. ${ }^{13-15}$ The HPS presents a correlation with the family history: $17 \%$ of risk in one member of the family, $3 \%$ in two or more members. ${ }^{16}$ In particular, if the father was affected by the condition, the risk of a child with the same condition is equal to $7 \%$; if the mother was the one affected, the risk increases 4 times. The incidence is 20 times greater for twins. The incidence of the condition varies according to the geographic location and the race. It is estimated that it ranges from $1.5-4$ cases per 1000 live births among Caucasians. ${ }^{12,17}$ The Afro-American and Asian population prove to be less affected. Males are more frequently affected than females with a ratio of 5:1.11 The symptoms appear between the 3rd and 6th week of life, rarely after the fourth month. In premature infants the onset is more insidious and occurs between the 32 nd and 87 th day of life. ${ }^{18}$ A late onset was also described. ${ }^{19,20}$ The clinical characteristics are onset of projectile non-bilious vomiting, after every feeding, representing the main symptom. Upon inspection, the gastric hyperperistalsis is noted with direction starting from below the left costal margin and disappearing right to the umbilicus, whereas, upon palpation, it possible to notice the olive-shape pyloric swelling. A late diagnosis and treatment might be associated with dehydration with important electrolyte imbalance (metabolic alkalosis and hypoproteinemia, hypochloremia, hyponatremia, hypokalemia and hyperazotemia). Clinically, these elements result in the interruption of weight gain, typical facies (sunken eyes and fontanelles), possible tetany. Hematemesis might sometimes occur due to the possible association with reflux gastritis or esophagitis. ${ }^{16}$

\section{Diagnosis}

A correct anamnesis and an accurate clinical examination allow to define a correct HPS diagnosis in $75-80 \%$ of cases, an ability that in the past years is getting lost.16,21 In 1986, Haller and Cohen reported and identified the following measurements detectable at the ultrasonography as reliable elements to diagnose the HPS: pyloric diameter $\geq 15 \mathrm{~mm}$, pylorus muscle wall thickness $\geq 4 \mathrm{~mm}$, pyloric canal length $\geq 18 \mathrm{~mm} .^{22}$ Since then, the ultrasonography has become increasingly more diffuse as a reliable device for HPS diagnosis thanks to the high specificity and sensitivity, the wide availability and the non-invasiveness, turning it into the preferred method of diagnosis. Forster et al. reported a sensitivity and specificity of the pyloric muscle wall thickness of $91 \%$ and $85 \%$, respectively, and a sensitivity and specificity of the pyloric muscle length of $76 \%$ and $85 \%$, respectively. ${ }^{23}$ Other authors reported similar results modifying the American measures of wall thickness $>3 \mathrm{~mm}$, pyloric diameter $>10 \mathrm{~mm}$ and pyloric canal length $>15 \mathrm{~mm} .{ }^{24}$ Three parameters are therefore taken into consideration for the ultrasonographic diagnosis of the HPS: the maximum transverse pyloric diameter; the pyloric canal length; the pyloric muscle thickness.

The normal pyloric thickness is approximately $2 \mathrm{~mm}$; a value greater than 3-4 mm is considered pathological.25,26 The transverse diameter is normally $<1 \mathrm{~cm}$. Values greater than or equal to $1.2 \mathrm{~cm}$ or $1.5 \mathrm{~cm}$ are considered pathological according to the clinical studies.

The length of the pyloric canal is included between 1.2 and 1.5 $\mathrm{cm}$ and it is considered pathological for values greater than 1.8 $\mathrm{cm} .{ }^{26,27}$

In HPS these measures are stable over time, helping the professional identify this condition. An indirect sign of the pyloric hypertrophy at the gastric level is described as the cervix sign as it resembles the uterine cervix in appearance. $22,28,29$ The ultrasound technique presents the following advantages: i) it does not expose the patient to radiations, ii) it avoids the risk of aspiration during the exam, iii) it allows to visualize the three dimensions of the pylorus, iv) the exam can be repeated. The disadvantages however are: i) difficult visualization of the stomach and the intestinal tract below the duodenum, ii) operator-dependent procedure. The methodology offers a sensitivity of $97 \%$ and a specificity of $100 \%$. In case of doubtful interpretation of the clinical and ultrasonographic criteria, it is possible to perform a contrast radiography of the upper gastrointestinal tract. The direct radiography of the abdomen can still be helpful today in the diagnosis of HPS in doubtful cases, allowing, at the same time, to rule out other possible conditions. The administration of contrast allows to identify some distinctive radiological patterns of the HPS: i) upward string deviation or string sign; ii) indentation of the base of the bulb or mushroom sign or umbrella sign; iii) spiked antrum (due to unequal hypertrophy of the muscle fasciae) or beak sign.22

\section{Indication for surgery}

The surgical treatment, nowadays, represents almost a forced choice for the HPS if compared to the conservative treatment for the following reasons: the high rate of success $(\sim 100 \%)$, the shortest length of hospital stays, the reduced rate of complications and a survival rate of almost $100 \%$.

The surgical therapy is never considered as a matter of urgency since, before the surgery, the complete stabilization and correction of the dehydration and the electrolyte imbalance is mandatory to avoid possible complications correlated with the anesthesia and the immediate postoperative phase. ${ }^{30}$ In case of greater hydroelectrolytic imbalance, it is fundamental to normalize the altered blood and chemistry levels. There is a wide variation in the HPS fluid and electrolytes substitution protocols, however the typical protocol includes the correction with a solution containing $0,45 \% \mathrm{NaCl}$ and $5 \%$ or $10 \%$ dextrose with $\mathrm{KCl}$ added 
to $10-20 \mathrm{mmol} / 500 \mathrm{ml}$. Some would indicate the integration with further saline boli in relation to the Chlorine concentration. However, there are no existing evidences confirming that one preoperative hydration regimen is superior to another. The monitoring of the electrolytes in this phase of rehydration remains fundamental. In literature, there are controversies regarding the necessity of positioning a nasogastric tube during the preoperative phase for the preoperative continuous gastric decompression, ${ }^{31}$ the use of prophylactic antibiotics 32,33 and the use of antacids. 34 The positioning of the nasogastric tube is however recommended as the patient is occluded. The antibiotic prophylaxis seems more indicated to reduce the risk of surgical wound infection.

\section{Surgical approach}

The extramucosal pyloromyotomy suggested by Dufour and Fredet since 1908 and modified by Dr. Conrad Ramstedt in 1912, "Fredet-Ramstedt extramucosal pyloromyotomy", represents the gold standard surgical treatment for the hypertrophic pyloric stenosis. $6,7,35$

In this surgical era, thanks to the increasingly greater development of the minimally invasive surgery in its different forms and variations, the classic open approach (right transverse supraumbilical minilaparotomy) in the right hypochondrium utilized to perform the extramucosal pyloromyotomy has been gradually substituted by new minimally invasive approaches (both open and laparoscopic) with optimal results as reported by the literature. $36-40$

Thanks to the high rate of success, the open transumbilical approach described by Tan and Bianchi in $1986^{41}$ and the videoassisted laparoscopic approach with three trocars described by Alain et al. in 19919,42 have reported a greater diffusion.

Furthermore, several randomized and controlled studies comparing these two techniques reported similar excellent results with no statistically significant differences in terms of complications. ${ }^{43-45}$

Therefore, we will focus the writing of our guidelines by taking into consideration mainly these two approaches.

However, in literature there are few publications describing the use of new techniques as the needlescopic approach, 46,47 the single-incision approach, ${ }^{48-50}$ the new endoluminal endoscopic pyloromyotomy and the balloon dilation. $51-53$ These procedures require special surgical abilities and further reports are necessary to evaluate the real advantages, rate of complications and possible contraindications. For these reasons, we do not intend to evaluate or include these new techniques in our guidelines.

\section{Patient preparation}

The patient has to be kept in a heated location, in the same way the operating room has to be conveniently heated before starting the surgery. In case of open approach, the patient is supine on the operating table, close to the right side in order to be closer to the first surgeon, with a small cushion positioned under the back to expose the superior abdominal region. In case of a laparoscopic approach, the position is the same as the one of the open approach even though the young patient might be positioned transversally on the operating table to facilitate the laparoscopic setting. It is recommended to position a nasogastric tube right before the surgical procedure to void and deflate the stomach and it can also be used to rule out the perforation of the mucosa during the pyloromyotomy. 54

\section{Open Approach (surgical technique)}

\section{Traditional approach}

The traditional approach described by Fredet-Ramstedt establishes an incision of approximately $3-4 \mathrm{~cm}$ at the right upper quadrant (right hypochondrium), 2-3 $\mathrm{cm}$ below the margin of the liver. 55 Access to the abdominal cavity with no section of the abdominal wall muscles (fibers diastasis). Opening of the peritoneum and access to the abdominal cavity. The liver is carefully moved upward, the stomach is identified then grasped and pulled with atraumatic forceps. Proceeding distally the pyloric olive is identified and exteriorized by means of a ring forceps (atraumatic) or manually with the help of moist gauzes. The traction is applied from the ending region of the gastric antrum proceeding distally with small, oscillating, accurate movements and without applying a maximal force. With the pylorus between the index and thumb of the left hand (right for left-handers), once identified its limits with the stomach and the duodenum, a longitudinal incision (along its major axis) is performed on the avascular antero-superior surface that, starting from the serosa, penetrates the superficial muscle thickness. The incision has to start at the level of the junction between the pylorus and the duodenum in correspondence with the right gastric vein (where, upon palpation, a feeling of a gap is appreciated) and it has to proceed until the gastric side, until the margin of the hypertrophic portion. Once the incision is performed, it is deepened by blunt dissection with a smooth-tip clamp. The maneuver, although delicate, allows the muscle fibers to split with minimal pressures for the entire thickness in order to favor the eversion and herniation (bulging) of the mucosa. Once the mucosa is identified, in order to better dissect the pyloric muscle fibers, the surgeon uses a special clamp called Ramstedt clamp. In case this clamp is not available, the maneuver can be performed with the smooth and rounded end of a scalpel handle, or with a smooth Kelly clamp with the tip pointing upward. Greater attention needs to be paid in the dissection near the duodenum. In absence of complications, once verified the efficacy of the myotomy, the pylorus is reintroduced in the abdomen without paying excessive attention to the possible exudate of the section margin. The wound is closed in layers and the skin is sutured intradermally.

\section{Tan and Bianchi transumbilical approach}

Over the last years, the transumbilical approach, described by Bianchi and Tan in 1986,41 has gained increasingly more diffusion thanks to the greater cosmetic result compared to the conventional right transverse incision lateral to the rectus muscle. 37,38

The technique requires the access to the pyloric region through an incision along the superior margin of the umbilical scar that allows to reach the abdominal cavity through the abdominal rectus muscles.

A supraumbilical or infraumbilical incision is made. The linea alba is sectioned longitudinally or transversely and the peritoneum is opened to access the abdominal cavity.

The pyloromyotomy can be performed via extracavitary or intracavitary technique:

i) The extracavitary technique consists of the exteriorization of the pylorus from the abdominal cavity lightly pulling the stomach with a Babcock/atraumatic clamp. An incision is performed on the anterosuperior side of the pylorus, starting from the junction between the pylorus and the duodenum approx. 2 $\mathrm{mm}$ proximally to the right gastric vein until the gastric antrum. The incision has to extend from the serosa to the 
superficial muscle fibers, followed by blunt dissection until obtaining the bulging of the mucosa with dissection of the muscle fibers via Ramstedt clamp or smooth tool (as above). In absence of complications, once verified the efficacy of the myotomy, the pylorus is reintroduced in the abdomen without paying excessive attention to the possible exudate of the section margin. The wound is closed in layers and the skin is sutured with 3-0 and 4-0 interrupted absorbable sutures.

ii) The intracavitary technique consists in grasping the pylorus with a Babcock clamp and moving it toward the incision without exteriorizing it from the abdominal cavity. Two sutures might be positioned in the hypertrophic muscles to lift and hold the pylorus in position. Then the pyloromyotomy is performed between the two sutures as described above. ${ }^{26}$ Eltayeb et al. ${ }^{56}$ reported statistically significant differences between the intracavitary and extracavitary technique in the operating time and in the average time of returning to oral feeding, at the same time no differences were observed related to the thickness of the pyloric muscle layer, to operative and postoperative complications, length of hospital stays and cosmetic results. Therefore, the intracavitary pyloromyotomy appeared safe and efficacious and more appropriate for large hypertrophic pylori without the need to widen the surgical wound.

The immediate complication that might occur is the perforation of the mucosa. In case the perforation is suspected, it is possible to rule it out by insufflating the stomach with air via the nasogastric tube and, at the same time, by moistening the pyloromyotomy with a minimal quantity of saline solution and verifying whether gas bubbles leaks.

Even if the absence of bubbles suggests a low suspect of mucosal compromise, this technique does not completely rule out the possibility of perforation. ${ }^{54}$ In case leak of gas bubble is verified, the double layer closure of the incision is performed (possible omental reinforcement) and a new myotomy is performed on the posterior margin. Then the pylorus is reintroduced in the abdomen. $37,57,58$

\section{Laparoscopic Approach}

The video-assisted laparoscopic pyloromyotomy, performed in 1990 in Limonge by Alain and Grosseau, was first described in 1991.59 From then to now, publications on the technique are found in literature underlying the safety and feasibility of the technique, the low rate of complications, the success of the technique and the excellent cosmetic result. 8,39

The procedure was the same as described and practiced in standard literature. The infant is given general anesthesia via endotracheal intubation. A nasogastric tube is positioned to empty the stomach.

\section{Patient position}

The position of the patient on the operating table can be traditional or transverse (Figure 1). With the first, the patient is supine with the First Surgeon at the end of the table, the monitor positioned at head end, the Assistant to the left or to the right according to the needs. 60

With the transverse position, the patient is supine, with an across the table position, allowing a better organization of the equipment and a better ergonomic position for the Surgeon and the Anesthetist. The monitor is positioned to the right of the operative table and the Surgeon is in front of it, with the Assistant to the left or to the right according to the needs (Figure 2).

\section{Surgical technique}

The umbilical incision in performed and the first $5 \mathrm{~mm}$ trocar is placed using an Open technique. A 4-5 mm 30-degree scope is introduced for a better orientation inside the abdominal cavity and for the visualization of the anterior surface of the pyloric olive. Induction of the pneumoperitoneum with insufflation of carbon dioxide is started at $0.1 \mathrm{~L} / \mathrm{min}$, gradually increased to $1 \mathrm{~L} / \mathrm{min}$ with target pressure kept between 4-6 mmHg to avoid possible hemodynamic and ventilatory complications. It is recommended to pay particular attention to carefully clean the insufflation tube with $\mathrm{CO} 2$ before connecting it to the trocar in order to eliminate the room air in the system.

Is advisable to put an absorbable 3-0 U-suture at the level of the fascia or the skin at the umbilical level to hold the umbilical trocar and to avoid change of position during the surgery. Two $3-$ $5 \mathrm{~mm}$ trocars are inserted under vision maintaining the triangulation. The introduction of the trocar can be facilitated by the incision with a scalpel blade. During the introduction, it is recommended that the operator compensates the push created by the introduction of the trocar with a counter-pressure of the wall (essential maneuver in neonates due to the fragility of the skin). Position of the two trocars: on the right, in the right lower quadrant/right flank; on the left, in the epigastric area, approx. $1 \mathrm{~cm}$ left of the midline (Figure 3). An atraumatic Babcock clamp is

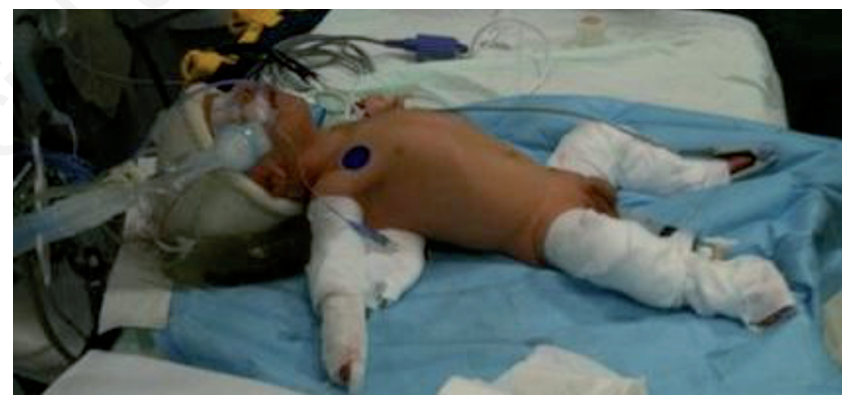

Figure 1. Transverse position for laparoscopic pyloromyotomy.

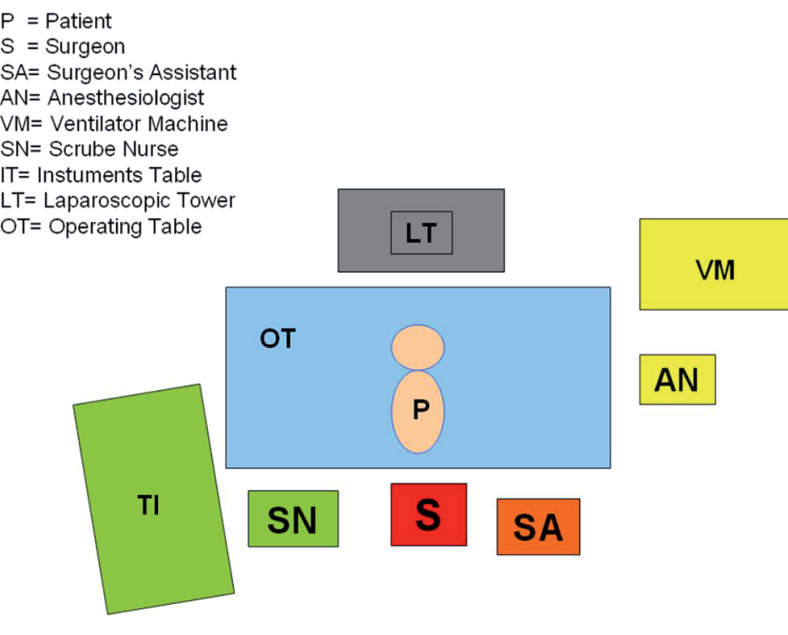

Figure 2. Operating-room set-up for laparoscopic pyloromyotomy. 
introduced in the trocar on the right flank, whereas the retractable scalpel or the scissors are introduced through the left trocar. It is often necessary to lift the inferior margin of the liver to visualize the pyloric olive (it is possible to place a traction suture at the level of the falciform ligament to expose the pyloric region). With the forceps, the pylorus is grasped in the middle (avoiding to clamp the duodenum, which is more delicate), it is slightly pulled and mobilized to expose its avascular anterosuperior surface. The scalpel blade is introduced through the left trocar and the incision of the serosa is performed from the center towards the duodenum (right gastric vein) until the origin of the gastric antrum. This incision can also be performed with scissors. The incision does not have to reach the mucosa in depth. Once the incision is performed, the scissors are pulled back and a spreader, known as pyloric spreader or an atraumatic clamp is inserted and the muscle margins are separated from the center towards the duodenum and towards the stomach until the herniation of the mucosa is obtained (Figure 4). It was also reported the use of a monopolar laparoscopic electrocautery hook (at very low energy) to perform the incision of the serosa and the muscle layers of the pylorus 61 (higher risk of mucosal perforation). A perforation test might be performed by dropping saline solution with a 3-5 mm laparoscopic irrigation system and by insufflating the stomach with air via a nasogastric tube inspecting for bubbles. Even though the absence of bubbles suggests a low suspect of mucosal compromise, this technique does not completely rule out a possible perforation. 54 The pneumoperitoneum is evacuated and the trocar incision are closed with 4-0 interrupted absorbable sutures. The skin might be closed with absorbable sutures or with glue. ${ }^{62}$

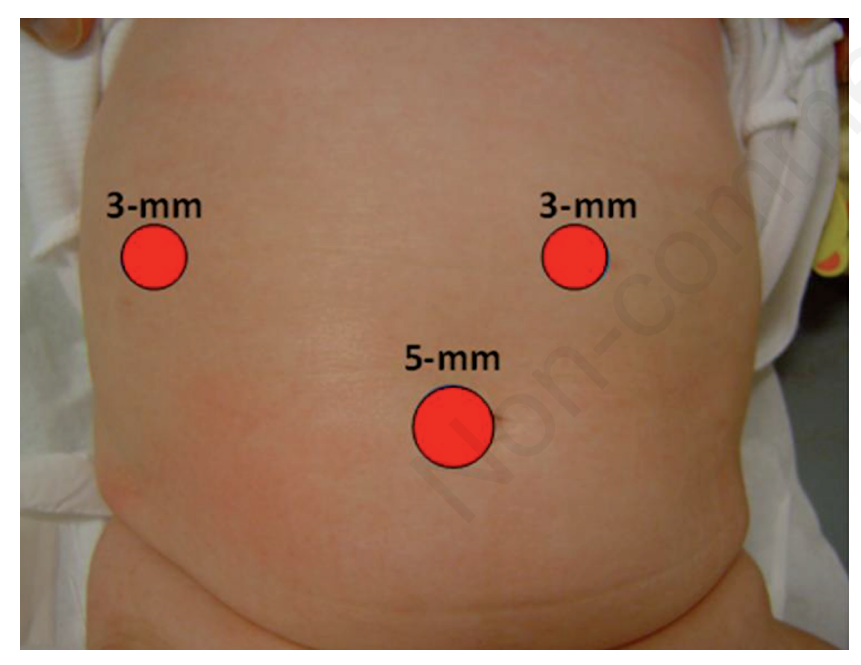

Figure 3. Trocar position for laparoscopic pyloromyotomy.
A variation of the technique was described with the use of an ophthalmic surgical knife on the left, introduced under vision without the use of trocar. The size of the blade used is $2.8 \mathrm{~mm}$ with a total length of $12 \mathrm{~cm}$, guaranteeing the persistence of the pneumoperitoneum by closing the wound with a purse string suture. With the use of this blade, the incision is performed and the pyloromyotomy is completed by dissecting the muscle fibers with a Benson pylori spreader introduced through the same incision inside the purse string suture. At the end of the Ramstedt pyloromyotomy the absence of mucosal lesions is verified, the instruments are removed under vision, the pneumoperitoneum is evacuated and the wounds are sutured. 63

\section{Postoperative management and outcome}

The intravenous fluid infusion is maintained until the patient returns to feeding according to the basic necessities. In the overwhelming majority of cases, the patient returns to oral feeding from 4 to 6 hours after surgery, first with a glucose solution, then with milk initially diluted (ratio of milk to water is $1: 1$ ), then with milk only, increasing the quantity to reach full feeds within 24-36 hours. Even if there are different protocols that regulate the volume increase and the feeds distribution in the reprisal of oral feeding, some support the ad libitum feeding with excellent results. ${ }^{64}$ The Table 1 shows a common protocol for oral feeding. The majority of the patients tolerate full feeds 24-48 hours after surgery and they might be discharged to home.65 Different

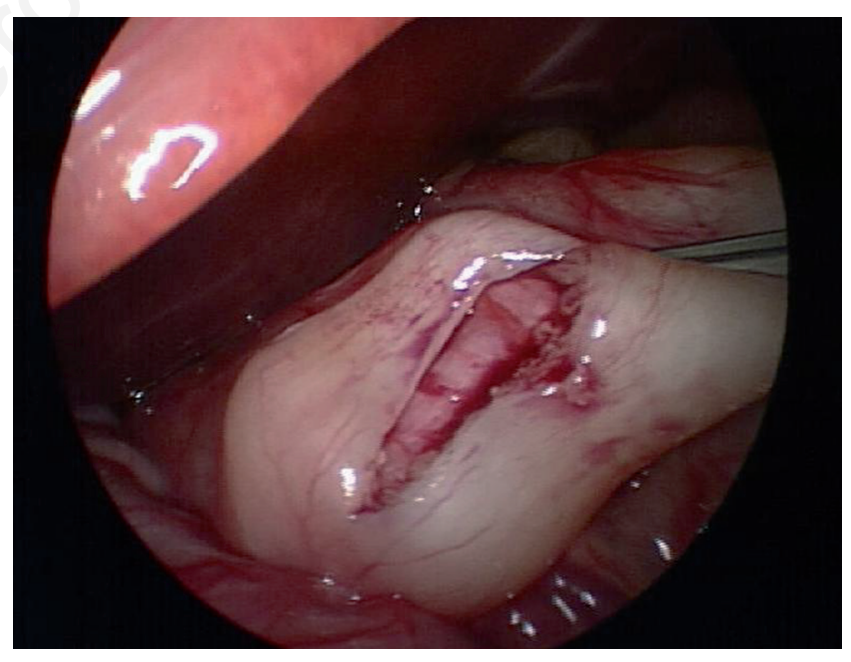

Figure 4. Laparoscopic view of pyloromyotomy.

Table 1. Feeding protocol 4-6 hours after pyloromyotomy.

\begin{tabular}{lcl} 
Sulbstance & Quantity & Time of interval \\
Pedialyte or water with 10\% glucose & $30 \mathrm{ml}$ & Every 3 hours \\
Half-strength formulaor complete breast milk & $30 \mathrm{ml}$ & Every 3 hours \\
\hline Full-strength formulaor breast milk & $30 \mathrm{ml}$ & Every 3 hours \\
Full-strength formulaor breast milk & $60 \mathrm{ml}$ & Every 3 hours \\
\hline Full-strength formulaor breast milk & $90 \mathrm{ml}$ & Every 3 hours \\
Full-strength formulaor breast milk & Ad libitum & Every 3 hours \\
\hline
\end{tabular}


researches compared the relation between the protocols for gradual reprisal of feeding, gradual precocious reprisal of feeding (before 4 hours) and ad libitum feeding, evaluating the relation with the frequency of vomiting episodes and the length of hospital stay. From a meta-analysis emerges that there are no correlations between the ad libitum feeding and the frequency of vomiting or the length of hospital stay. In fact, what emerges is that the timing of the first feed after surgery, either precocious (before 4 hours after surgery) or late (after 6 hours), does not determine the outcome. However, it is possible to deduce that a precocious reprisal of feeding is associated to a shorter hospital stay. ${ }^{66}$ Nevertheless, an ad libitum feeding status post Open or Laparoscopic pyloromyotomy, after the complete awakening following anesthesia, appears safe and reduces the necessary length of time to reach full feeds, reducing the length of hospital stay and therefore the costs without determining an increase in the rate of complications or readmission after the discharge from the hospital. Furthermore, there are few works in literature that analyze long-term follow-up status post pyloromyotomy. The outcomes of the HPS status post pyloromyotomy were studied in 103 neonates by Muramori et al. ${ }^{24}$ Some standard ultrasonographic measures were taken to evaluate the canal length, the muscle thickness and the pylorus diameter for a period of 1 year after the surgery. In contrast with the rapid improvement of symptoms, the ultrasound findings showed that the pyloric canal reached its normal length $(\sim 12,7 \pm 2,8 \mathrm{~mm})$ approximately 4 months after surgery, the muscle thickness reached a normal interval $(\sim 2 \mathrm{~mm})$ within 8 months, whereas the pylorus diameter $(\sim 10-12 \mathrm{~mm})$ did not normalized even 1 year after the pyloromyotomy. Walker at al. ${ }^{67}$ analyzed the neurological development of infants who underwent surgery due to HPS comparing it with a control group (healthy infants) at one year of age, noticing how the cognitive and receptive language and the motion score were significantly lower for the infants suffering from HPS compared to the control group. Other authors reported chronic abdominal pain most likely secondary to irritable bowel syndrome, functional dyspepsia and functional abdominal pain in infants who underwent HPS surgery during an average follow-up period of 7 years compared to the control group of healthy infants. ${ }^{68,69}$ These results raised concerns and discussions on the potential impact of the HPS and its surgical treatment. However, these are preliminary studies on the bases of which it is not possible to reach an adequate scientific conclusion.

\section{Other surgical techniques}

\section{Trocarless laparoscopic pyloromyotomy with traditional instruments}

The technique is performed with the introduction of a $5-\mathrm{mm}$ trocar in the umbilical region, the pneumoperitoneum is induced with insufflation starting from $0,1 \mathrm{~L} / \mathrm{min}$ and reaching $1 \mathrm{~L} / \mathrm{min}$. The pneumoperitoneum pressure is kept to $6 \mathrm{mmHg}$ and can fluctuate to a maximum of $8 \mathrm{mmHg}$, if needed. Two $3 \mathrm{~mm}$ instruments are then introduced through the abdominal wall incisions, 1 at the level of the right anterior axillary region above the umbilicus to grasp the stomach, the other in the epigastric region, above the pylorus, to incise the pylorus and separate the muscle fibers. The stomach is pulled towards the left to visualize the pylorus. Before starting each procedure, the hook insulation is verified. An incision is performed along the thickened pylorus from the stomach to the duodenum by using a 3-mm hook with monopolar electrical current of
30 Watts. The incision is then deepened. In case the coagulating electrical current is not adequate, it is possible to use the electrical current hook in the low voltage cutting mode (30 Watts). The muscle fibers are then separated with a $3 \mathrm{~mm}$ Maryland scissors until it is possible to visualize the herniation of the mucosa. An adequate pyloromyotomy is confirmed by the bulging of the mucosa and the presence of two separated pyloric edges. The perforation of the mucosa is ruled out by insufflating the stomach with air via the nasogastric tube. ${ }^{70-73}$

\section{Single-site pyloromyotomy}

This technique consists of a video-assisted laparoscopic pyloromyotomy using only a single laparoscopic port, with umbilical access, wider than the norm, with a particular trocar through which the 5-mm scope and two 3-mm instruments are inserted through two fascial incisions at the level of the umbilical region. The approach is transperitoneal and it repeats the same stages previously described. The advantages consist in the use of the umbilical scar only, avoiding unaesthetic small scars caused by the use of additional trocars. The duration of the surgery is reportedly short. The technique was used more frequently in the last years and it is defined as safe and efficacious. The disadvantages consist in the need of using dedicated instruments produced for this specific use only and by the fact that this technique requires a significant practical experience but above all a greater difficulty in the organ dissection (longer duration of surgery). ${ }^{74,75}$

\section{Endoscopic Balloon Dilation}

This technique was introduced in the treatment of HPS over the last decades. It is considered a safe procedure in the treatment of the idiopathic infantile hypertrophic pyloric stenosis and it is recommended as a starting approach before performing the pyloromyotomy. 76,77 The recurrent pyloric stenosis is rare in patients suffering from HPS after balloon dilation. ${ }^{76}$ However, since the endoscopic dilation does not break the hypertrophic muscular ring, ${ }^{78}$ it is preferably earmarked for patients with a significant anesthesiological and surgical risk. 79

\section{Video-assisted needle incision of the serosa and the muscle}

This procedure consists of the video-assisted laparoscopic percutaneous introduction of a $18 \mathrm{G}$ cannula utilized for the incision of the serosa and the superficial muscle layer ( 6 patients have been treated with this technique between January 2012 and September 2015). Since the body part of the needle is protected by a holder, this avoid the laceration of the mucosa and the incision is performed with a set cutting depth. 80

\section{Endoscopic pyloromyotomy associated with balloon dilation}

Few cases of association of this technique are described in particular for patient that cannot undergo surgery. 81 


\section{Single-port, laparoscopic-assisted pyloromyotomy}

The access to the abdominal cavity is performed through a right circumferential umbilical incision positioning a $12-\mathrm{mm}$ trocar. After inducing the pneumoperitoneum, an operative optic is introduced. Once the pylorus is located, it is easily localized and then grasped and exteriorized through umbilical access. At this point, conventional Ramstedt pyloromyotomy is performed. Once the pylorus is reintroduced into the abdomen, a new pneumoperitoneum is created to check mucosal integrity and hemostasis. ${ }^{82}$

\section{Complications}

Controlled randomized studies, by comparing the open and laparoscopic approach for the Fredet-Ramstedt pyloromyotomy, demonstrated that there are no statistically significant differences in terms of complication rate. $9,43-45$

The rate of operative or postoperative complications is low, generally correlated to the incomplete experience of the operating surgeon in the different phases of the learning curve. 40,69

The most frequent complications are the perforation of the mucosa and the incomplete pyloromyotomy. In both cases, the treatment requires a second surgery performed by an "expert" surgeon and it can be performed both with an open or laparoscopic approach.

The perforation of the mucosa mainly occurs after an excessive dissection of the muscle fibers, in particular on the duodenal side of the pylorus. In this case, the perforation is closed with an absorbable suture covered with an omental patch. Later, a new pyloromyotomy can be performed on the posterosuperior side of the pylorus.

The incomplete pyloromyotomy is secondary to a short inci- sion or a non-adequate partial separation of the muscle fibers on the gastric side of the pylorus. In this case it is necessary to perform a new surgery to complete the pyloromyotomy.

Other more serious and very rare complications have already been signaled as the carbon dioxide embolism during the laparoscopic pyloromyotomy. $70-72$

\section{Conclusions}

The introduction of ultrasound brought important changes in the diagnosis of HPS. A prompt diagnosis, the stabilization and correction of the dehydration and the electrolyte imbalance allow the young patient to undergo the surgical correction at favorable conditions and with better outcomes. From 1912 to now, the Fredet-Ramstedt extramucosal pyloromyotomy represents the gold standard.

The review of controlled randomized studies in literature that compare the surgical procedure with Open and Laparoscopic approach shows that both approaches are equally safe and reproducible in expert hands. From a cosmetic point of view, there are no real benefit between the transumbilical open approach and the laparoscopic approach. The surgeon has to opt for the best approach according to the personal experience, the own surgical abilities and the resources of the institution where he/she works (Figure 5). Moreover, a close supervision of an Expert Pediatric Surgeon is compulsory and fundamental during the learning path of the training medical doctors in order to reduce the risk of major complications.

Further studies on new techniques such as the ones described above are necessary to evaluate their real benefits, complications and contraindications in this field.

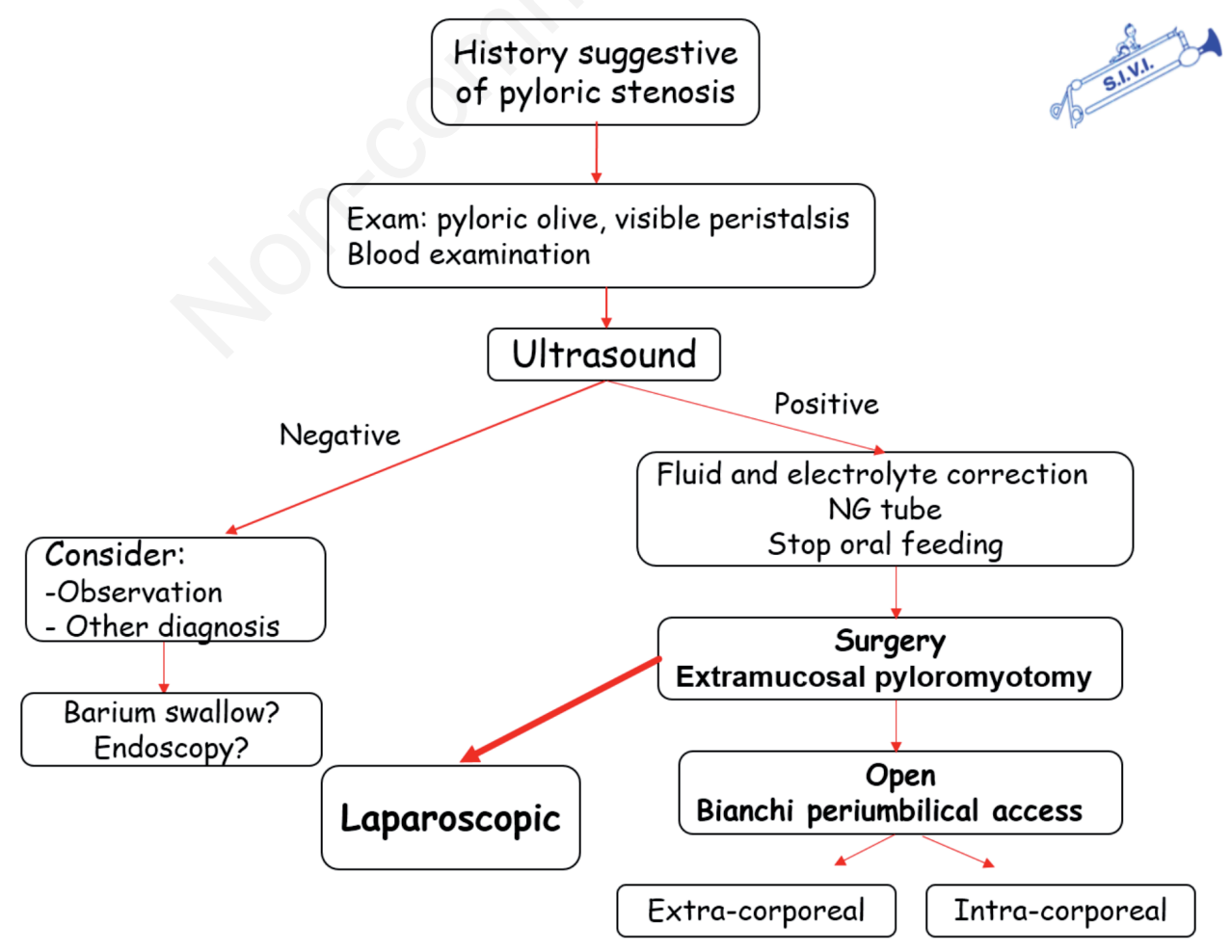

Figure 5. HPS Flow Chart Management. 


\section{References}

1. Takeuchi M, Yasunaga H, Horiguchi H. et al. Pyloromyotomy versus i.v. atropine therapy for the treatment of infantile pyloric stenosis: nationwide hospital discharge database analysis. Pediatr Int 2013;55:488-91.

2. Luak M, Antunovic SS, Vulovic D, et al. Is abandonment of nonoperative management of hypertrophic pyloric stenosis warranted? Eur J Pediatr Surg 2013;23:80-4.

3. Aspelund G, Langer JC. Current management of hypertrophic pyloric stenosis. Semin Pediatr Surg 2007;16:27-33.

4. Hildanus F. Opera omnia. Frankfurt, J. Bayerns; 1646.

5. Hirschsprung H. Fälle von angeborener Pylorusstenose, beobachtet bei Säuglingen. Jb. Kinderheilk 1888;28:61-68.

6. Dufour H, Fredet P. La sclerose hypertrophique du pylore chez le nourisson et son traitment chirurgical. Rev Chir 1908;37:208

7. Shaw A. Ramstedt and the centennial of pyloromyotomy. J Pediatr Surg 2012;47:1433-5.

8. Sola JE, Neville HL. Laparoscopic vs open pyloromyotomy: a systemic review and meta-analysis. J Pediatr Surg 2009;44:1631-7.

9. Oomen MWN, Hoekstra LT, Ubbink DT, et al. Open versus laparoscopic pyloromyotomy for hypertrophic pyloric stenosis: a systematic review and meta-analysis focusing on major complications. Surg Endosc 2012;26:2104-10.

10. Serra A, Schuchardt K, Genuneit J, et al. The role of RET genomic variants in infantile hypertrophic pyloric stenosis. Eu J Pediatr Surg 2011;21:389-94.

11. Krogh C, Gortz S, Wohlfahrt J, et al. Pre-and perinatal risk factors for pyloric stenosis and their influence on the male predominance. Am J Epidemiol 2012;176:24-31.

12. Pedersen RN, Garne E, Loane M, et al. Infantile hypertrophic pyloric stenosis: a comparative study of incidence and other epidemiological characteristics in seven European regions. J Matern Fetal Neonatal Med 2008;21:599-604.

13. Hauben M, Amsden GW. The association of erythromycin and infantile hypertrophic pyloric stenosis: causal or coincidental? Drug Saf 2002;25:929-42.

14. Lozada LE, Royall MJ, Nylund CM, et al. Development of pyloric stenosis after a 4-day course of oral erythoromycin. Pediatr Emerg Care 2013;29:498-9.

15. Murchison L, De Coppi P, Eaton S. Post-natal erythromycin exposure and risk of infantile hypertrophic pyloric stenosis: a systematic review and meta-analysis. Pediatr Surg Int 2016;32:1147-52.

16. Taylor ND, Cass DT, Holland AJ. Infantile hypertrophic pyloric stenosis: has anything changed? J Paediatr Child Health 2013; 49:33-7.

17. Chan SM, Chan EKW, Chu WCW, Cheung ST, Tam YH, Lee $\mathrm{KH}$. Hypertrophic pyloric stenosis in a newborn: a diagnostic dilemma. Hong Kong Med J 2011;17:245-7.

18. Cook M. Gastric outlet obstruction. In: Lister J, Irving I. Neonatal surgery (ed.) Butterworth \& Co. London; 1990.

19. Boybeyi O, Karnak I, Ekinci S, et al. Late-onset hypertrophic pyloric stenosis: definition of diagnostic criteria and algorithm for the management. J Pediatr Surg 2010;45:1777-83.

20. Mahalik S, Prasad A, Sinha A, et al. Delayed presentation of hypertrophic pyloric stenosis: a rare case. J Pediatr Surg 2010;45:e9-11.

21. Forman HP, Leonidas JC, Kronfeld GD. A rational approach to the diagnosis of hypertrophic pyloric stenosis; do the results match the claims? J Pediatr Surg 1990;25:1149-50.
22. Haller JO, Cohen HL. Hypertrophic pyloric stenosis: Diagnosis using US. Radiol 1986;161:335-9.

23. Forster N, Haddad RL, Choroomi S, et al. Use of ultrasound in 187 infants with suspected infantile hypertrophic pyloric stenosis. Australas Radiol 2007;51:560-3.

24. Muramori K, Nagasaki A, Kawanami T. Ultrasonographic serial measurements of the morphologic resolution of the pylorus after Ramstedt pyloromyotomy for infantile hypertrophic pyloric stenosis. J Ultrasound Med 2007;26:1681-7.

25. O'Keeffe FN, Stansberry SD, Swischuk LE, Hayden CK. Antropiloric muscle thickness at US in infants: what is normal. Radiol 1991;178:827-30 .

26. Reed AA, Michael K. Hypertrophic pyloric stenosis. J Diagn Med Sonography 2010,26:157-60.

27. Blumhagen JD, Maclin L, Krauter D, et al. Sonographic diagnosis of hypertrophic pyloric stenosis. AJR Am J Roentgenol 1988; 150:1367-70 .

28. Costa Dias S, Swinson S, Torrão H, et al. Hypertrophic pyloric stenosis: tips and tricks for ultrasound diagnosis. Insights Imaging 2012;3:247-50.

29. Hernanz-Schulman M, Sells LL, Ambrosino MM, et al. Hypertrophic pyloric stenosis in the infant without a palpable olive: accuracy of sonographic diagnosis. Radiol 1994;193:771-6.

30. Wilkinson DJ, Chapman RA, Owen A, et al. Hypertophic pyloric stenosis: predicting the resolution of biochemical abnormalities. Pediatr Surg Int 2011;27:695-8.

31. Elanahas A, Pemberton J, Yousef Y, et al. Investigating the use of preoperative nasogastric tubes and postoperative outcomes for infants with pyloric stenosis: a retrospective cohort study. J Pediatr Surg 2010;45:1020-3.

32. Mullassery D, Perry D, Goyal A, et al. Surgical practice for infantile hypertrophic pyloric stenosis in the United Kindom and Ireland: a survey of members of the British Association of Pedaitric Surgeons. J Pediatr Surg 2008;43:1227-9.

33. Katz MS, Schwartz MZ, Moront ML, et al. Prophylactic antibiotics do not decrease the incidence of wound infections after laparoscopic pyloromyotomy. J Pediatr Surg 2011;46:1086-8.

34. Banieghbal B. Rapid correction of metabolic alkalosis in hypertrophic pyloric stenosis with intravenous cimetidine: preliminary results. Pediatr Surg Int 2009;25:269-71.

35. Raveenthiran V. Centennial of Pyloromyotomy. J Neonat Surg 2013;2:8

36. Gauderer MW. Experience with a non laparoscopic, transumbilical, intracavitary pyloromyotomy. J Pediatr Surg 2008;43:884-8.

37. Lazar D, Naik B, Fitch ME, et al. Transumbilical pyloromyotomy with umbilicoplasty provides ease of access and excellent cosmetic results. J Pediatr Surg 2008;43:1408-10.

38. Cozzi DA, Ceccanti S, Mele E, et al. Circumbilical pyloromyotomy in the era of minimally invasive surgery. J Pediatr Surg 2008;43:1802-6

39. Lange R, Rey M, Fernandez ED. Open vs. laparoscopic pyloromyotomy- a retrospective analysis. Minim Invasive Ther Allied Technol 2008;17:313-7.

40. Oomen MWN, Hoekstra LT, Baky R. Learning curves for pediatric laparoscopy: how many operations are enough? The Amsterdam experience with laparoscopic pyloromyotomy. Surg Endosc 2010;24:1829-33.

41. Tan KC, Bianchi A. Circumumbilical incision for pylo- romyotomy. Br J Surg 1986;73:399.

42. Alain JL, Grousseau D, Terrier G. Extramucosal pyloromyotomy by laparoscopy. Surg Endosc 1991;5:174-5. 
43. Nigel JH, Van der Zee J, Hock LT, et al. Meta-analysis of laparoscopic versus open pyloromyotomy. Ann Surg 2004;240:774-8.

44. St. Peter SD, Holcomb GW, Calikins CM, et al. Open versus laparoscopic pyloromyotomy for pyloric stenosis. A prospective, randomized trial. Ann Surg 2006;244:363-70.

45. Siddiqui S, Heidel RE, Angel CA, et al. Pyloromyotomy: randomized control trial of laparoscopic versus open technique. J Pediatr Surg. 2012;47:93-8.

46. Turial S, Enders J, Schier F. Microlaparoscopic pyloromyotomy in children: initial experiences with a new technique. Surg Endosc 2011;25:266-70.

47. Turial S, Enders J, Schier F, et al. Comparison of a novel technique of the microlaparoscopic pyloromyotomy to circumbilical and Weber-Ramstedt approaches. J Gastrointest Surg 2011;15:1136-42.

48. Muensterer OJ, Adibe OO, Harmon CM, et al. Single-incision laparoscopic pyloromyotomy: initial experience. Surg Endosc 2010;24:1589-93.

49. Muensterer OJ, Chong AJ, Georgeson KE, et al. The crosstechnique for single-incision pediatric endosurgical pyloromyotomy. Surg Endosc 2011;25:3414-8.

50. Kozlov Y, Novogilov V, Podkamenev A, et al. Single-incision laparoscopic surgery for pyloric stenosis. Pediatr Surg Int 2012;28:347-50.

51. Ibarguen-Secchia E. Endoscopic pyloromyotomy for congenital pyloric stenosis. Gastrointest Endosc 2005;61:598-600.

52. Zhang YX, Nie YQ, Xiao X, et al. Treatment of congenital hypertrophic pyloric stenosis with endoscopic pyloromyotomy. Zhonghua Er Ke Za Zhi 2008;46:247-51.

53. Hsun-Chin Chao. Update on endoscopic management of gastric outlet obstruction in children. World J Gastrointest Endosc 2016;8:635-45.

54. Lee SL, Sydorak RM, Lau ST. Air insufflations of the stomach following laparoscopic pyloromyotomy may not detect perforation. JSLS 2010;14:60-1.

55. Benson C.: Infantile pyloric stenosis, historical aspects and current surgical concepts. Pr Ped Surg 1970;1:63.

56. Eltayeb AA, Othman MH. Supraumbilical pyloromyotomy: a comparative study between intracavitary and extracavitary techniques. J Surg Educ 2011;68:134-7.

57. El-Gohary Y, Yeap BH, Hempel G, et al. A 9-year single center experience with circumumbilical Ramstedt's pyloromyotomy. Eur J Pediatr Surg 2010;20:387-90.

58. Emil S. Pyloromyotomy through an infra-umbilical incision: open technique and superb cosmesis. Eur J Pediatr Surg 2009;19:72-5.

59. Alain JL, Grousseau D, Terrier G. Extramucosal pyloromyotomy by laparoscopy. J Pediatr Surg 1991;26:1191-2.

60. Tej Handu A, Jadhav V, Deepak J, et al. Laparoscopic pyloromyotomy: Lessons learnt in our first 101 cases. J Indian Assoc Pediatr Surg 2014;19:213-7.

61. Jain V, Choudhury SR, Chadha R, et al. Laparoscopic pyloromyotomy: is a knife really necessary? World J Pediatr 2012;8:57-60.

62. Binet A, Klipfel C, Meignan P, et al. Laparoscopic pyloromyotomy for hypertrophic pyloric stenosis: a survey of 407 children. Pediatr Surg Int 2018;34:421-6.

63. Anwar MO, Al Omran Y, Saeed Al-Hindi S. Laparoscopic
Pyloromyotomy: A Modified Simple Technique. J Neonatal Surg 2016;5:3.

64. Juang D, Adibe OO, Laituri CA, et al. Distribution of feeding styles after pyloromyotomy among pediatric surgical training programs in North America. Eu J Pediatr Surg 2012;22:409-11.

65. Lee SL, Stark R. Can patient factors predict early discharge after pyloromyotomy? The Permanente J 2011;15:44-6.

66. Sullivan KJ, Chan E, Vincent J, et al. Feeding PostPyloromyotomy: A Meta-analysis. Pediatrics 2016;137: e20152550.

67. Walker K, Halliday R, Holland AJ, et al. Early developmental outcome of infants with infantile hypertrophic pyloric stenosis. J Pediatr Surg 2010;45:2369-72.

68. Saps M, Bonilla S. Early life events: infants with pyloric stenosis have a higher risk of developing chronic abdominal pain in childhood. J Pediatr 2011;159:551-4.

69. Haricharan RN, Aprahamian CJ, Celik A, et al. Laparoscopic pyloromyotomy: effect of resident training on complications. J Pediatr Surg 2008;43:97-101.

70. Taylor SP, Hoffman GM. Gas embolus and cardiac arrest during laparoscopic pyloromyotomy in an infant. Can J Anaesth 2010;57:774-8.

71. Fischler M. Carbon dioxide embolism in a 3-week-old neonate during laparoscopic pyloromyotomy: a case report. J Pediatr Surg 2009; 44:1864.

72. Kudsi OY, Jones SA, Brenn BR. Carbon dioxide embolism in a 3-week-old neonate during laparoscopic pyloromyotomy: a case report. J Pediatr Surg 2009;44:842-5.

73. Parelkar S.V, Multani P.V, Sanghvi B.V, et al. Trocarless laparoscopic pyloromyotomy with conventional instruments: Our experience. J Minim Access Surg 2013;9:159-62.

74. Li B, Chen WB, Wang SQ, Wang YB. Single-site umbilical laparoscopic pyloromyotomy in neonates less than 21-day old. Surg Today 2015;45:29-33.

75. Harmon CM. Single-site umbilical laparoscopic pyloromyotomy. Semin Pediatr Surg 2011;20:208-11.

76. Nasr A, Ein SH, Connolly B. Recurrent pyloric stenosis: to dilate or operate? A preliminary report. J Pediatr Surg 2008;43:e17-e20.

77. Ogawa Y, Higashimoto Y, Nishijima E, et al. Successful endoscopic balloon dilatation for hypertrophic pyloric stenosis. J Pediatr Surg 1996;31:1712-4.

78. Hayashi AH, Giacomantonio JM, Lau HY, Gillis DA. Balloon catheter dilatation for hypertrophic pyloric stenosis. J Pediatr Surg 1990;25:1119-21.

79. Hsun-Chin Chao. Update on endoscopic management of gastric outlet obstruction in children. World J Gastrointest Endosc 2016;8:635-45

80. Bataineh ZA, Novotny NM. A Novel Nonelectrosurgical Technique for Incising the Pylorus in Laparoscopic Pyloromyotomy. J Laparoendosc Adv Surg Tech 2017;28:235-6.

81. Yokoyama S, Uyama S, Iwagami H, Yamashita Y. Successful combination of endoscopic pyloromyotomy and balloon dilatation for hypertrophic pyloric stenosis in an older child: A novel procedure. Surg Case Rep 2016;2:145.

82. Bertozzi M, Prestipino M, Nardi N, Appignani A. Preliminary experience with a new approach for infantile hypertrophic pyloric stenosis: the single-port, laparoscopic-assisted pyloromyotomy. Surg Endosc. 2011;25:2039-43. 